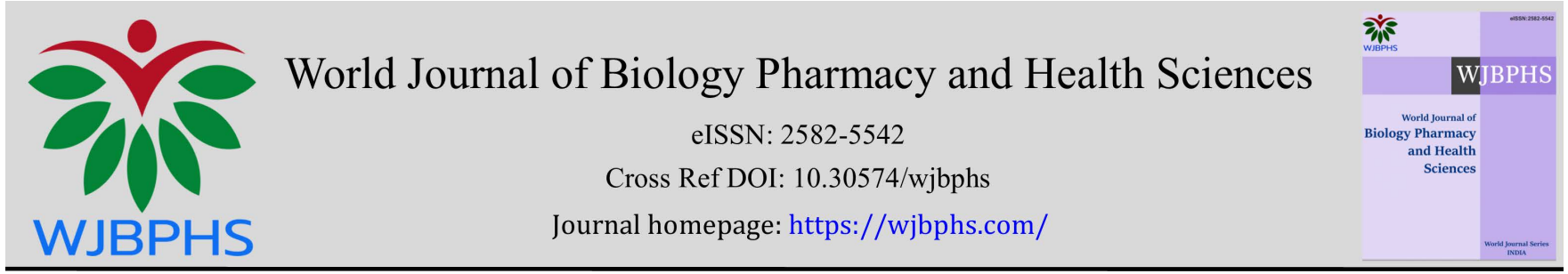

(REVIEW ARTICLE)

\title{
Hydrogels for management of chronic wound healing
}

\author{
IM Cardoso-Daodu *, CP Azubuike and MO Ilomuanya \\ Department of Pharmaceutics and Pharmaceutical Technology, Faculty of Pharmacy. University of Lagos, PMB 12003, \\ Surulere, Lagos, Nigeria.
}

World Journal of Biology Pharmacy and Health Sciences, 2021, 05(03), 095-104

Publication history: Received on 12 February 2021; revised on 14 March 2021; accepted on 17 March 2021

Article DOI: https://doi.org/10.30574/wjbphs.2021.5.3.0027

\begin{abstract}
Chronic wounds occur when one wound healing process or a sequence of wound healing events are affected resulting in slow healing of the wound thereby placing the patient in deep pain. Various diseases and conditions can delay the process of wound healing. Wound healing can be classified into four main stages: hemostasis, inflammation, remodeling, and scar tissue formation with each phase overlapping one another. The skin is the largest organ in the body. It protects the entire external surface of the human body and is the primary site of interaction with the outside environment. There is therefore a need to fabricate an ideal dressing through scientific research and investigations.

Hydrogels are a three-dimensional network of hydrophilic polymers that can swell in water and absorb copious amounts of water while maintaining their structure because of their chemical or physical crosslinking of individual polymer chains. A hydrogel must be composed of at least $10 \%$ water. Hydrogels possess the flexibility and water percentage which is remarkably like tissues. They are biocompatible and biodegradable which makes them ideal for dermal wound healing.
\end{abstract}

Keywords: Cell; Wound; Healing; Tissue; Hydrogel

\section{Introduction}

Chronic wounds occur when one of the wound healing processes or multiple events are affected resulting in slow healing of the wound thereby placing the patient in deep pain [1]. Various diseases and conditions can delay the process of wound healing. Wound healing can be classified into four main stages: hemostasis, inflammation, remodeling, and scar tissue formation with each phase overlapping one another.

Homeostasis or coagulation, which is the first phase of wound healing begins at the onset of the injury. Homeostasis involves vasoconstriction and blood clotting which aims to prevent blood loss, it also creates a provisional matrix for cell division. Growth factors are secreted by platelets cytokines which allow the presence of fibroblast while immune and endothelial cells start the healing process. The inflammation phase lasts about one week. Phagocytic cells are primarily at work in this phase. Neutrophils and macrophages work hand in hand to further the healing process. Neutrophils provide reactive oxygen species (ROS) and proteases that guard against bacterial contamination. It works to also cleanse the wound bed of cellular debris [2].

The proliferative stage is dominated by tissue granulation, epithelization, and angiogenesis. The objective of the proliferative stage is to reduce the wound area by contraction and fibroplasia. This stage is responsible for the closure of the wound with levels that include angiogenesis, fibroplasia, and re-epithelialization. The proliferative phase begins

\footnotetext{
${ }^{*}$ Corresponding author: I M Cardoso-Daodu

Department of Pharmaceutics and Pharmaceutical Technology, Faculty of Pharmacy. University of Lagos, PMB 12003, Surulere, Lagos, Nigeria.
}

Copyright (C) 2021 Author(s) retain the copyright of this article. This article is published under the terms of the Creative Commons Attribution Liscense 4.0. 
two days after the wound has occurred and is in process for at least two weeks. Remodeling of the vessels to improve flow, endothelial cell proliferation, and realignment of the basal membrane also occur in the proliferative stage [3].

The fourth phase of healing which is the remodeling of scar tissue formation begins twenty-one days after healing begins and can last up to two years. The objective of the remodeling stage is to return the skin to the maximum tensile strength through reorganization, degradation, and resynthesis of the extracellular matrix. An alteration on any of the healing stages may lead to an extension in the time phase of the wound healing process. This allows for the wound surface to degenerate to a chronic state. Chronic wounds are largely characterized by bacterial and fungal infections. There are a variety of underlying conditions that can lead to slow wound healing thereby resulting in a chronic state. Treatment of these underlying conditions is recommended and where necessary cessation of negative habits or medications [4].

\subsection{Underlying Health Conditions}

Conditions such as diabetes, rheumatoid arthritis, jaundice, fibrosis, vascular aethereal diseases, hemophilia, bacterial and fungal infections, poor nutrition, and zinc deficiency. All of which alter the pattern or phases and expected time of cutaneous healing.

\subsection{Medicines}

Drugs that affect wound healing can be classified. Antineoplastic, Anticoagulants, Corticosteroids, and Non-steroidal anti-inflammatory drugs.

\subsection{Habits}

Alcohol addiction and Smoking.

Wound care can be defined as the promotion of wound healing through topical wound care. This may start with the cleansing of the wound. Care must be taken to ensure that fluid used to clean wound such as ringers or physiologically saline solutions are at body temperature when utilized. Application of medicaments such as antibacterial topical creams such as silver sulphadiazine or gentian violet should come next. Finally, the dressing of the wound is carried out. A few basic things should be considered in the choice of a wound dressing. First, it must be of a reasonable cost, must be able to fit into body contours, should be designed in such a way that dress changing is less traumatic, it should control moisture, and allow for airflow [5].

Recently modern dressings are being fabricated to fit this description. However, most dressings do not fit into all the requirements. Alginate, activated carbon, hyaluronic acid, hydrogel, impregnated gauze, collagen, and honey are all examples of modern wound dressings. These are all attempts at an ideal dressing however some more research needs to be done on them to achieve the perfect dressing.

Hydrogels are three-dimensional polymer network structures that can absorb copious amounts of water. Hydrogels occur naturally as polymer structures. Collagen and gelatin are ideal examples of natural hydrogels. The polymeric structure of hydrogels composed mainly of water is held together by covalent crosslinks, ionic forces, hydrogen bonds, affinity bio-recognition polymer crystallites, and physical entanglement of polymer chains. Hydrogels have been extensively used in the treatment of wounds for the past ten years. To accelerate chronic wound healing many hydrogels have been formulated. Natural or synthetic hydrogels have the unique ability to absorb and control moisture at the site of the wound. They are physiologically compatible and biodegradable. Hydrogels can be synthetic, natural, or hybrid (a mixture of synthetic and natural). Smart ideas and innovations in the field of hydrogels for chronic wound healing have been on-going and have resulted in novel fabrications such as pH-sensitive, temperature-sensitive, electro-sensitive, and light-sensitive hydrogels for wound dressing.

\section{The skin}

The skin is the largest organ in the body. It protects the entire external surface of the human body and is the primary site of interaction with the outside environment. The complex structure of the skin and its biochemical properties make it an effective outermost defense against exogenous factors. The primary physicochemical parameters for characterizing the state of the skin are $\mathrm{pH}$, epidermal hydration, trans- epidermal water loss, and sebum excretion. The skin partakes in the process of metabolism. It also plays a role in resorption, temperature regulation, and immunological functions. The skin also is the primary line of defense against pathogenic micro-organisms, toxins, ultraviolet-radiation, trauma, chemicals, and mechanical injury. The skin's thickness depends on the region of the body and is grouped based 
on the thickness of the epidermal and dermal layers. The palms of our hands and soles of our feet are the thickest. The skin generally is made up of 15-20 layers of cornified keratinocytes called corneocytes [6].

\subsection{Epidermis}

The epidermis does not contain any blood vessels and is completely dependent on the dermis for nutrient and waste disposal by diffusion through the dermo-epidermal layer. The epidermis is a stratified squamous epithelium that consists primarily of keratinocytes in the progressive stages of differentiation from deeper to more superficial layers [7]. Keratinocytes manufacture proteins =are known as keratin. It contains melanocytes which produce skin pigment known as melanin. Langerhans cells protect the skin from foreign substances [8]. The layers of the epidermis include -

\begin{tabular}{ll} 
- & Stratum basale \\
- & Stratum spinosum \\
- & Stratum granulosum \\
- & Stratum lucidum \\
\hline & Stratum corneum
\end{tabular}

\subsubsection{Stratum basale}

It is also known as stratum geminativum and is the innermost layer. A basement membrane separates it from the dermis. It is attached to the basement membrane by the hemidesmosomes, cells. Cells found in this layer are cuboidal to columnar mitotically active stem cells that are constantly producing keratinocytes.

\subsubsection{Stratum spinosum}

Stratum spinosum is a Latin term which translates spinous layer. This layer is found right in between the stratum basale and stratum granulosum. It has 8-10 cell layers and can also be called the prickle layer. It contains irregular polyhedral cells with cytoplasmic processes named spines. These spines extend outward and are in contact with neighboring cells by desmosomes. The dendritic cell can also be found in this layer. Its keratinocytes are polyhedral with big pale staining nuclei. Actively synthesizing fibrillary proteins are important for the creation of desmosomes. The desmosomes attach the cells and tightly connect the cells. Keratinization starts in this layer. Langerhans cells are also found in the stratum spinosum [9].

\subsubsection{Stratum granulosum}

Stratum granulosum means granular layer. This layer is composed of diamond-shaped cells with keratohyalin granules and lamellar granules. It has about five cell layers and is located between the stratum corneum or stratum lucidum and the stratum spinosum. The keratinocytes in the stratum granulosum are specifically granular. These keratinocytes are distinctively referred to as granular cells. They are composed of keratohyalin which helps in the binding of keratin from one filament to another. Keratinocytes have lamellar bodies filled with lipids which are secreted into the extracellular space through exocytosis. This leads to the formation of a lipid barrier. These keratinocytes secrete them and move from the stratum spinosum to the upper layers of the epidermis. Eventually, cells lose nuclei and organelles and become corneocytes when they reach the stratum corneum [10].

\subsubsection{Stratum lucidum}

It is made up of 2-3 cell layers. It is part of the thicker skin found in the palms and soles of the feet. Stratum lucidum is a Latin term which means transparent layer. Its name is gotten from its translucence under the microscope. Its presence in the hand and feet reflect the necessity of having thicker skin to shield against mechanical stress. Stratum lucidum is made up of keratinocytes which are filled with eleidin which is later converted to keratin [11].

\subsubsection{Stratum corneum}

After stratum lucidum, the keratinocytes go into the next layer called the stratum corneum. It is a Horney layer filled with cornified cells. The corneocytes form a hard protective barrier that shields against environmental trauma, abrasions, light, heat, chemicals, and micro-organisms. The corneocytes finally shed themselves into the environment and mites readily feed on them. A complete cycle from new keratinocyte in the stratum basale to dead cell flaked off into the air take between 25-45 days. 


\subsection{Dermis}

The dermis is also known as the corium. It is the thicker inner layer of the skin under the epidermis. It is composed of connective tissue. The dermis is attached to the epidermis through the basement membrane and has two layers of connective tissue.

\section{- The papillary layer}

- The reticular layer

The papillary layer is near the skin surface it can be as wide as 300-400 microns depending on its age and location. The upper part is arranged into cords named the dermal papillae with nerve endings and microvascular vessels important for nourishment and innervation. The papillary layer has a higher density of cells compared to the reticular layer more proteoglycans and a weaker arrangement of collagen fibers. It has an unlevelled polar structure its density decreases as we move from the basement membrane to the reticular layer [12].

The reticular layer is set apart from the papillary layer by the vascular plexus and the rete sub-papillae. The extracellular matrix of the reticular dermis is more complex than that of the papillary layer. Collagen bundles are arranged into dense fibers together with elastin strands to make an organized mesh. An increase in age leads to a decrease in the papillary volume and thickness, it is gradually replaced by the reticular dermis [13].

\subsection{Hypodermis}

The hypodermis is composed of loose connective tissues made up of fat. They protect and cushion the skin. Fibroblast adipose cells and macrophages are particularly found in the hypodermis. These cells have a role to play in tissue remodeling and foster thermogenesis of fat during exposure to cold and stress. Adipocytes are arranged in form of lobules. The hypodermis plays a vital role in adipose hemostasis and is populated with G-protein-coupled receptors. The basement part of the hypodermis is devoid of fat and composed mainly of fibers. The hypodermis is also made up of microvacuolar tissue and stores up interstitial fluid. Tissue swelling is modulated by the fibrous components of the hypodermis. The viability of the microvacuolar tissue is vital for fluid distribution and hemostasis.

\section{Chronic wounds}

Chronic wounds are wounds that are unable to progress through the set of stages of healing. Healing cannot occur in an orderly and prompt manner. The healing process is not complete and prevented by some factors which prolong one or more stages in various pathways. Factors that affect the phases of wound healing include infection, tissue hypoxia, necrosis, exudate, excess levels of inflammatory cytokines. A perpetual condition of inflammation allows for a line of tissue responses that create a non-healing state. Due to the unorganized manner of healing the injured tissue or skin is unable to restore to $90 \%$ normalcy, with poor tissue scarring and the possibility of wound relapse. Chronic wounds can be grouped into three vascular ulcers. The diabetic ulcer, vascular ulcer, and pressure ulcer. They all manifest a prolonged inflammatory phase, chronic infections, development of drug-resistant microbial colonies, lack of response to reparative stimuli by the skin. A combination of these manifestations leads to the final chronic state of the wound [14, 15].

\section{Phases of chronic wound healing}

\subsection{Coagulation and Hemostasis Phase}

Injury leads to an outpour of blood and lymphatic fluid, the coagulation and hemostasis phase set in. Production of the reparative coagulum occurs. Intrinsic and extrinsic clotting mechanisms are activated. Also, thrombocytes are produced, and vasoconstriction occurs. After vasoconstriction, platelets adhere to damaged endothelium and release ADP adenosine diphosphate leading to thrombocyte clumping which blocks the wound surface. Vasoconstriction is stopped, vessels are dilated allowing the inflow of thrombocytes and other blood cells [16].

\subsection{Inflammatory Phase}

The inflammatory phase begins immediately after hemostasis is achieved. The main aim of this stage is to get rid of pathogens and microbial colonies and contain the wound to the wound-bed. There is an increase in vascular permeability through vasodilation thereby accumulating neutrophils and monocytes at the wound bed. The interference of cytokines helps to co-ordinate this phase resulting in monocyte conversion to macrophages. The macrophages digest debris and excess neutrophils they also secrete growth factors and cytokines that combine to enhance tissue 
proliferation and cellular migration. After 72 hours of the onset of the original wound, the proliferative stage based on fibroblast production of collagen will form the basis for the tissue scaffold.

\subsection{Proliferation or Granulation Phase}

This phase consists of overlapping subphases namely, fibroplasia, matrix deposition, angiogenesis, and reepithelization. Within a week of the onset of the wound, healing fibroblast has migrated into the wound bed attracting new collagen of subtypes I and III. Type III collagen predominates initially but is later replaced by type I collagen.

Tropocollagen which is a precursor to collagen is converted to collagen by the cell's endoplasmic reticulum. Proline and lysine are also hydroxylated by the rough endoplasmic reticulum. Three tropocollagen strands form a triple left-handed helix through disulfide bonds, this left-handed helix is called procollagen. The release of procollagen into the extracellular space causes peptidases in the cell wall to cleave terminal peptide chains creating collagen fibrils [4].

The wound is permeated by glycosaminoglycans and fibronectin produced by fibroblast. The glycosaminoglycans are composed of heparin sulfate, hyaluronic acid, chondroitin sulfate, and keratan sulfate. Angiogenesis commences the formation of new vasculature this requires the extracellular matrix and basement membrane decomposition, seconded by migration, mitosis, maturation of endothelial cells. Angiogenesis is modulated by the fibroblast growth factors and vascular endothelial growth factor.

Migration of the cells within the wound bed leads to re-epithelization of the wound. The primary factor at this stage of wound healing is the epidermal growth factor. Those phases can last a maximum of four weeks in a clean uninfected wound [17].

\subsection{The Remodeling Phase}

This is the final phase of wound healing it includes the elimination of the granulation tissue. The epidermis dermal vasculature nerves and myofibers of the skeletal muscles are renewed creating functional tissue. Vascular components of fibroblast and myofibroblast of the granulation tissue are reduced. Peripheral blood mononuclear cells are subjected to apoptosis or migrate away from the wound bed. The concentration levels of glycosaminoglycans that enabled structural recovery and hydration are eliminated. Degradation and deposition of collagen in a feedback equilibrium manner occurs maintaining the quantity of collagen in the wound. Collagen type III is degraded and replaced with collagen type I which gives parallel fibrils and low cellularity scar. Contraction occurs at the wound surface. The tensile strength of the wound occurs at week twelve which may be up to $90 \%$ of the original tensile strength before the occurrence of the wound $[18,19]$.

\section{Factors that affect chronic wound healing}

\subsection{Underlying diseases}

\subsubsection{Diabetes}

Wound healing is a complex and serious process that can be classified into four stages: hemostasis, inflammation, proliferative, and remodeling phases. Each stage is overlapping the other, these stages involve many cell types, extracellular components, growth factors, and cytokines. Diabetes Mellitus leads to poor wound healing through the alteration of mechanisms and pathways it acts by causing hyperglycemia, chronic inflammation, micro, and macro circulatory dysfunction, hypoxia, autonomic and sensory neuropathy. In chronic wound healing, the process of healing is delayed in the inflammatory cell accumulation resulting in alterations of growth factor expressions. Growth factors affect wound healing and act to catalyze the process of wound healing. Growth factors influence the process through an inhibitory and stimulatory effect on the wound bed. A group of growth factors is present in wound healing with the most important growth factors being: PDGF, PGF, VEDF, EGF, NGF, GM-CSF [14; 20].

A poor expression of these growth factors (which is the case with diabetes mellitus) leads to poor wound healing.

\section{PDGF (Platelet-derived growth factor)}

PDGF plays a primary role in the wound healing process. It acts as a mitogen on fibroblasts, vascular smooth muscle cells, endothelial cells, neurons, and macrophages and schematic agents for neutrophil plays an important role in the wound healing process, its addition to wound bed catalyzes wound healing. 


\section{FGF (Fibroblast growth factor)}

It is known to enhance cell proliferation and regulate the movement and differentiation of cells of mesodermal, ectodermal, and endodermal origin. FGF is mitogenic for different cell types present at the wound bed including fibroblast and keratinocytes.

\section{EGF (Epidermal growth factor)}

This growth factor plays a vital role in re-epithelialization and granulation tissue formation. It is mitogenic for fibroblast and keratinocytes.

\section{VEGF (Vascular Endothelial Growth Factor)}

It is a primary regulator of both vasculogenesis, and angiogenesis and its levels get higher during trauma and ischemia. VEGF also serves to enhance wound angiogenesis.

\section{NGF (Nerve growth factor)}

NGF is important for the growth and survival of certain sympathetic and sensory neurons both in the central and peripheral nervous system.

\section{GM-CSF (Granulocyte-macrophage colony stimulation factor)}

GM-CSF takes part in angiogenesis and mitogenic for keratinocytes. GM-CSF is also vital in the cytokine role in inflammatory diseases. Due to the pro-cellular effect, a lack of balance of GM-CSF secretion and signaling leads to dangerous inflammatory conditions [21].

\subsubsection{Rheumatoid arthritis}

Rheumatoid arthritis is a systemic autoimmune condition that is known to have environmental factors and is more frequent in females and the geriatric demographic, its presents with inflammation of the joint and erosion of the bone. It has episodes of activity along with periods of remission during which signs and symptoms of the disease are absent. Rheumatoid Arthritis is thought to have a genetic component and is also influenced by environmental factors such as viruses and smoking.

Overtime rheumatoid arthritis has been connected to the occurrence of wounds and delay of wounds. Rheumatoid arthritis which is systematically inflammatory affects smaller joints of the hands and feet, it can also occur on the wrist and knees. Inflammation of the synovial membrane within the joints results in strain, immobility, swelling, and pain. Medications used to treat rheumatoid arthritis led to impaired wound healing. Disease-modifying anti-rheumatic drugs act on the immune system to stop the radiological progression of rheumatoid arthritis. Adversely they have a hindering impact on the inflammatory and proliferative phases of wound healing.

Management of wounds in rheumatoid arthritis requires a multi-disciplinary approach. Preventive health management includes, monitoring of disease-modifying rheumatic drugs and required screening for vasculitis in other organs for the body and sepsis by a doctor. Treatment also requires the engagement of a physio or occupational therapist to ensure mobility of joints and a nurse that can advise on emollient therapy [22].

\subsubsection{Bacterial and fungal infection}

At the onset of a wound, micro-organisms gather at the surface of the wound to access tissues within. The degree of infection determines whether a wound is having contamination, colonization, local infection, or invasive infection. Contamination is when there is the presence of organisms in the wound, but the organisms do not multiply. Colonization is the presence of micro-organisms that multiply without any damage to the structure and the tissues of the wound. Local infection is an intermediate stage of micro-organism reproduction and the beginning of tissue response. Invasion infection is defined as the presence of multiplying micro-organism inside the wound bed with injury to the structure and tissues of the wound. The infection eventually leads to prolonged inflammation. Bacteria like P. aeruginosa, E. coli, and fungal species Candida spp. majorly dominate the microbial populace [23]. 


\subsubsection{Zinc deficiency}

Zinc is an important trace element and a micronutrient that plays a necessary role in the human body system. Zinc is necessary for human wellbeing because of its critical roles in growth, development, bone metabolism, central nervous system, immunity, and wound healing. Some proteins are indispensable in cellular roles such as transcript regulation, DNA repair, metabolic processing, cellular matrix regulation, apoptosis, and antioxidant defense. The presence of zinc in the body and its regulation is an important part of cell structure. Zinc is particularly important to the skin, exactly $5 \%$ of zinc is found in the skin primarily the epidermis. Due to its high content in the epidermis, mildly low deficiency of zinc leads to roughened skin and impaired wound healing. Zinc deficiency is caused by malnutrition. Illness or infections and high phytate-containing foods prevent the availability of zinc. Wound healing, inflammation, and immune response and deeply associated with one another. Zinc lowers immune response through a multitude of pathways. Zinc promotes platelet activity and aggregation which leads to the initiation of the inflammatory phase. Zinc deficiency has important impact $\mathrm{T}$ lymphocyte populations. Regulatory $\mathrm{T}$ lymphocytes regulate and suppress inflammation. Zinc deficiency increases oxidative stress as oxidative damage is one of the causes of tissue injury [24].

\subsection{Drugs}

\subsubsection{Steroids}

Steroids significantly inhibit most aspects of wound healing. They delay the presence of inflammatory cells, fibroblast delivery of collagen, and regeneration of capillary through contraction and epithelial migration. In a study by Wicke et al, methylprednisolone was seen to markedly decrease TGF- $\beta$ and IGF- 1 levels. This reflects that corticosteroids have inhibitory effects on growth factors and collagen availability in the process of wound healing [25].

\subsubsection{NSAID'S}

Non-steroidal anti-inflammatory drugs have an inhibitory effect on wound healing as it decreases granulocytic inflammatory reaction. NSAID's prevent the production of PGE2 an inflammatory mediating prostaglandin that alleviates pain. The suppression of PGE2 is part of the process of wound scarring. NSAIDs may increase scar formation, particularly if used in the proliferative stage of wound healing. NSAIDs exhibit anti-proliferative properties in the veins, arteries, dermis, and epidermis. The effect of NSAIDs on wound healing includes a noticeable reduction in fibroblast which leads to the prevention of proliferation. This reflects that whilst NSAIDs are good for pain relief from wounds, they may slow the overall wound healing process. This also shows that NSAID's should be used with care in patients that have wounds [26].

\subsection{Habits}

\subsubsection{Alcohol}

Alcohol affects all organs and tissues in the body on a cellular and molecular level. Alcohol changes physiological function by direct interaction with cellular components. Alcohol metabolism affects the oxidative, immune function, and inflammatory state of the wound. Metabolism of alcohol leads to the production of toxic metabolites that affect normal cellular function needed for wound repair. Wound repair is made up of three overlapping phases that are in control of a variety of cellular functions with the final point being $90 \%$ healing back to normal [27].

\subsubsection{Smoking}

Chronic wounds create a lot of economic and social burdens globally. Chronic wounds may be caused or worsened by several conditions such as age obesity, diabetes mellitus, and habits such as smoking. Cigarette smoking impacts wound healing negatively in different areas. Patients who smoke and have chronic wounds are encouraged to stop.

Nicotine is an opaque alkaloid that is fast absorbed during smoking, physiologically it leads to increased blood viscosity, low oxygen levels, and poor wound healing. Carbon monoxide which is a by-product of smoking also causes hypoxia. It reduces oxygen by forming a complex with hemoglobin. Hydrogen cyanide which is also a by-product affects the process of tissue metabolism. Nicotine replacement agents are available in form of inhalers, patches, gums, nasal, sprays, and lozenges [28].

\section{Hydrogels for chronic wound healing}

Hydrogels encompass a group of materials that are vastly used in soft tissue engineering of the skin, blood vessel muscle, and fat. Hydrogels are three-dimensional meshes composed of physically or chemically cross-linked bonds of 
hydrophilic polymers. They have a peculiar potential to absorb moisture and exudates at the surface of the wound and allow oxygen diffusion thereby facilitating chronic wound healing.

Essentially hydrogels possess a very hydrated 3D polymeric mesh and can bind several folds more water in comparison to their normal weight before absorption, so they can regulate the high moisture environment of the wound bed. As a result of its peculiar physical properties hydrogel meshes can be molded into different shapes and sizes. Hydrogels also allow for the loading of cells, medicaments, growth factors, and biomacromolecules.

Hydrogels have a remarkable similarity to the extracellular matrix, therefore, provide a cell-friendly 3D environment that allows for tissue regeneration with or without cells embedded in a scaffold. Hydrogels satisfy the main requirement of biocompatibility for inpatient pharmacological use. They also have the physical and mechanical attributes suited for wound healing.

A hydrogel is a three-dimensional network of hydrophilic polymers that can swell in water and absorb copious amounts of water while maintaining its structure because of its chemical or physical crosslinking of individual polymer chains. Hydrogel must be composed of at least $10 \%$ water. The crosslinking of hydrogels with water provides and maintains mechanical rigidity and physical structure. Hydrogels can absorb water up to 10-20 times their molecular weight and become swollen [29].

\section{Types of hydrogels for chronic wound healing}

Hydrogels offer the great opportunity of incorporating medicinal or bioactive compounds within their polymer mesh and release them in a controlled manner at the site of action. Hydrogels can be fabricated to address the primary problems of chronic wound healing such as infection and inflammation. Based on the composition novel hydrogels can be sensitive to the wound environment such as $\mathrm{pH}$-sensitive, temperature-sensitive, electrosensitive, and lightresponsive hydrogels. These smart hydrogels are targeted at aiding the biochemical processes and pathways of angiogenesis that are responsible for the structural healing of the chronic wound site [30].

\subsection{Temperature-sensitive hydrogels}

Temperature-sensitive hydrogels are made up of both hydrophobic and hydrophilic hydrogel structures. Change in temperature leads to a change in the reaction between the hydrophilic and hydrophobic parts of the temperaturesensitive hydrogel. The temperature sensitivity of the hydrogel is derived from an equilibrium between the hydrophobic and hydrophilic portions of the polymer network. As the temperature is lowered the hydrogen bonds of the polymer framework and water molecules become strengthened resulting in dissolution in water. On the other hand, if the temperature is elevated reactions with hydrophobic portions become stronger and hydrogen bonds are weakened causing gelling. The temperature of a chronic wound bed is between 31-35 ${ }^{\circ} \mathrm{C}$. An increase in temperature from room temperature $21^{\circ} \mathrm{C}$ to wound temperature leads to gelling and allows the hydrogel to absorb moisture and swell. Moisture control is essential at the wound site as moisture encourages microbial growth [30].

\subsection{Near-infrared light-responsive hydrogels}

Near-infrared light-responsive hydrogels are subjected to structural alterations when irradiated with light of relevant wavelength. Alteration in chemical structures and polymeric functional groups leads to the expansion and release of loaded drug molecules. Changes are coordinated by light-sensitive polymeric functional groups known as chromophores. The chromophore absorbs the radiation and transforms it to heat thereby elevating the intrinsic temperature of the hydrogel. This leads to the expansion of the hydrogel and the release of loaded drug content [30].

\section{3. pH-sensitive hydrogels}

pH-sensitive hydrogels can swell when the $\mathrm{pH}$ of the surrounding environment is altered. They have acidic and basic portions that adjust to the changes in the $\mathrm{pH}$ of the environment by the addition or loss of hydrogen ions. $\mathrm{pH}$-sensitive hydrogel swelling is because of ionic hydrogels possessing charged pendant functional groups. Factors such as pKa or $\mathrm{pKb}$, level of ionization hydrophilicity polymer concentration, and $\mathrm{pH}$ of the environment. The swelling of hydrogels in contact with water relies on osmotic pressure within the polymer network of the hydrogels [30].

\section{Conclusion}

Hydrogels show great potential as wound dressings. They possess the vital properties of an ideal wound dressing which include the control of moisture at the site of the wound through absorption of wound exudate, alleviation of pain at the 
wound site by the cooling effect of the hydrogel polymer, and finally, the ability of the hydrogel to act as cargo for bioactive drug molecules that actively participate in the wound healing process [31].

\section{Compliance with ethical standards}

\section{Acknowledgments}

The author would like to thank Professor. Boladale Silva for his helpful comments and discussions.

\section{Disclosure of conflict of interest}

There is no conflict of interest between the authors of this publication.

\section{References}

[1] Han G, Ceilley R. Chronic Wound Healing: A Review of Current Management and Treatments. Advances in Therapy. 2017; 34(3): 599-610.

[2] Frykberg R, Banks J. Challenges in the Treatment of Chronic Wounds. Advances in wound care. 2015; 4(9): 560582.

[3] Guo S, DiPietro L. Factors affecting wound healing. Journal of Dental Research. 2010; 89(3): 219-229.

[4] Kolter J, Feuerstein R, Zeis P, Hagemeyer N, Paterson N, d'Errico P, Baasch S, Amann L, Masuda T, Lösslein A, Gharun K, Meyer-Luehmann M, Waskow C, Claus-Werner F, Grün D, Lämmermann T, Prinz M, Henneke, P. A Subset of Skin Macrophages Contributes to the Surveillance and Regeneration of Local Nerves, Immunity. 2019; 50(6): 1482-1497.

[5] Velnar T, Bailey T, Smkolj V. The Wound Healing Process: An Overview of the cellular and Molecular Mechanism. The Journal of International Medical Research. 2009; 37(5): 1528-1942.

[6] University of Leeds. Skin Layers of the Epidermis [Internet]. England, University of Leeds. 2020 [cited 2021 Jan 29]. Available from https://histology.leeds.ac.uk/skin/

[7] Baroni A, Buommino E, De Gregorio V, Ruocco E, Ruocco V, Wolf R. Structure, and function of the epidermis related to barrier properties. Clinical Dermatology. 2012. 30(3):257-262.

[8] Yousef H, Alhajj M, Sharma S. Anatomy, Skin (Integument), Epidermis. Treasure Island, Florida: StatPearls Publishing. 2020.

[9] Gonzalez C, Costa F, Andrade A, Medrado R. Wound healing - A literature review. An Bra Dermatol. 2016; 91(5): 614-620.

[10] Amirlek, B., Shahabi, L. Skin Anatomy [Internet]. United States of America, Medscape. 2017 [cited 2021 Jan 28]. Available from https://emedicine.medscape.com/article/1294744-overview

[11] Lumen. Anatomy and physiology boundless. Layers of the epidermis [internet]. Lumen Candela. 2020.

[12] Rippa L, Kalabusheva P, Vorotelyak A. Regeneration of Dermis: Scarring and Cells Involved. Cells. 2019; 8(6): 607-37.

[13] Wong R, Geyer S, Weninger W, Guimberteau C, Wong K. The dynamic anatomy and patterning of skin. Experimental Dermatology. 2016; 25(2): 92-98.

[14] Baltzis D, Eleftheriadou I, Veves A. Pathogenesis, and treatment of impaired wound healing in diabetes mellitus: new insights. Advances in Therapy. 2014; 31(8): 817-36.

[15] Thana N, Demidova R, Hamblin M, Herman I. Acute and Impaired wound healing: Pathophysiology and current methods for drug delivery. Advances in skin and wound care. 2012; 25(7): 304-314.

[16] Mescher A. Macrophages and fibroblasts during inflammation and tissue repair in models of organ regeneration. Regeneration. 2017; 4(2): 39-53.

[17] Mercandetti M. Wound Healing and Repair [online] Medscape. 2021.

[18] Cañedo-Dorantes L, Cañedo-Ayala M. "Skin Acute Wound Healing: A Comprehensive Review. International Journal of Inflammation. 2019; 1-15. 
[19] Schindeler A, McDonald M, Bokko P, Little G. Bone remodelling during fracture repair: The cellular picture. Semin Cell Dev Biol. 2008; 19(5): 459-66.

[20] Thanh D, Elder S, Veves A. Delayed wound healing in diabetes considering future treatments. Review. Future Medicine Diabetes Management. 2011; 135(11): 1265-70.

[21] Noushin L, Rodolfo T, Nahid R, Guang-Xian Z, Abbas R, Abdolmohamad R, Nafiseh E. Roles of GM-CSF in the Pathogenesis of Autoimmune Diseases: An Update. Frontiers in Immunology. 2019; 10(1265): 1-14.

[22] Young T. Rheumatoid arthritis and its impact on ulceration and healing. Wounds. 2019; 15(4): 40-43.

[23] Kalan L, Grice A. Fungi in the Wound Microbiome. Advances in Wound Care New Rochelle. 2018; 7(7): 247-255.

[24] Lin P, Sermersheim M, Li H, Lee P, Steinberg S, Ma J. Zinc in Wound Healing Modulation. Nutrients. 2017; 10(16): 1-20.

[25] Wicke C, Halliday B, Allen D, Roche N, Scheuenstuhl N, Spencer M, Roberts B, Hunt T. Effects of steroids and retinoids on wound healing. Archives of Surgery. 2000; 135(11): 1265-70.

[26] Anderson K, Hamm L. Factors That Impair Wound Healing. The Journal of American College Clinical Wound Specialists. 2014; 4(4): 84-91.

[27] Jung K, Callaci J, Lauing K, Otis J, Radek K, Jones K, Kovacs J. Alcohol exposure and mechanisms of tissue injury and repair. Alcoholism Clinical and Experimental Research. 2011; 35(3): 392-399.

[28] McDaniel C, Browning, K. Smoking, chronic wound healing, and implications for evidence-based practice. Journal Wound Ostomy Continence Nursing. 2014; 41(5): 415-423.

[29] de Sousa Moraes P, Saska S, Barud H, de Lima L, Martins V, Pepsi A, Ribeiro S, Gaspar A. Bacterial Cellulose/Collagen Hydrogel for Wound Healing. Materials Research. 2016; 19(1): 106-16.

[30] Chatterjee S, Hui P. Stimuli-Responsive Hydrogels: An Interdisciplinary Overview. In: Lacramioara P, Mihaela V, Elena C, Privu D. Intech Open. 2018; 1-23.

[31] Koehler J, Bradl F, Goepferich A. Hydrogel wound dressing for bioactive treatment of acute and chronic wounds. European Polymer Journal. 2018; 100(1): 1-11. 